doi: 10.17492/manthan.v2i2.8610

\title{
New Paradigm of Infrastructure Services - Concerns and Issues Relating To Public Private Partnerships
}

\author{
Sunita Sharma*
}

\begin{abstract}
India today is plagued by inadequate infrastructure that struggles to keep up with an ever increasing population. This study highlights the importance of a new approach to infrastructure investment, through commercialization of infrastructure projects. This research paper attempts to study the international experience of Public Private Partnership (PPPS) in infrastructure programs and why India needs widespread use of PPPs and various public private initiatives in India. We also look at the performance of top fifteen companies that have adopted the PPP format. In addition, this paper identifies factors constraining private investment in the infrastructure sector, and gives suggestions for future rollout of PPPs.
\end{abstract}

Keywords: Public Private Partnerships, Non-Performing Assets, Engineering Procurement and Construction, Total Project Cost, Viability Gap Funding.

\subsection{Introduction}

Infrastructure plays a key role in increasing economic growth. Various studies have found that infrastructure has a positive impact on output, especially in developing countries. India today is plagued by inadequate infrastructure that struggles to keep up with an ever increasing population. Infrastructure has been a relatively neglected sector till the end of the X Five Year Plan. Inadequate infrastructure was recognised in the XI Plan, as a major constraint on rapid growth. India needs to create infrastructure to the extent of $\$ 10$ trillion in three decades. Infrastructure development requires the services of firms that specialize in engineering, procurement and construction. Public Private Partnership (PPPs) projects will be the order of the day in future. Already several public limited companies in the private sector have shown their interest in entering this field.

Professor and Head of the Department, Department of Commerce, Maniben Nanavati Women's College, Mumbai. 


\subsection{Objectives of the study}

The study has been carried out with the following objectives:

- To review the international experiences of private participation in infrastructure programs through PPP schemes and its impact on infrastructure development.

- To explore why India needs widespread use of PPPs and various public private initiatives in India.

- To analyse the performance of top fifteen companies that have adopted PPP format, so as to determine the present and future environment for PPPs.

- To find out the factors constraining private investment in the infrastructure sector.

- To list the suggestions for future rollout of PPPs.

\subsection{Research methodology}

This paper has taken secondary data. The ratio analysis had been used. The parameters used for analysis are - profitability, rate of return and ability to repay debt. An analysis of top fifteen infrastructure companies, that have adopted PPP format have been analysed.

\subsection{International Experiences}

Numerous countries in Latin America and the Caribbean are moving towards a second phase of private participation in infrastructure programs - mostly through public - private partnership schemes. Several concerns remain from the outcomes of the first phase. Andres et al (2008) give the most comprehensive analysis to date of the impacts of private participation as well as the determinants of those impacts. The authors recap the main findings and suggest key corrections to move forward. From the accumulated experience of more than twenty years of PPP programs, the authors have suggested for policy makers the critical elements to be introduced in moving forward to secure success and maximum benefits of private partnership programs. By attacking the problems and deficiencies of the past, through second - generation reforms that are constructive and participatory and designing new processes that incorporate lessons learned, the improved performance of infrastructure sector would be achieved.

Marin's (2009) book is directed at policy makers in governments as well as donors. The study analyses the access, service quality, operational efficiency, and tariff levels of more than sixty five large water PPP projects, oven fifteen years in different regions. The objective of their study is to contribute to a better understanding of how to 
tackle the challenges of providing water and sanitation services to urban population in the developing world. The results achieved by many projects in service and efficiency improvements reaffirm the value of PPPs.

An examination of the available empirical evidence shows that well designed partnerships between the public and private sectors are a valid option to turn around poorly performing water utilities in developing countries. Emphasis should shift from trying to attract private investment, and using private operators to improving services quality and efficiency. The utility improves its financial situation and gradually, is able to finance a large share of its investment needs. Although concessions have worked in a few places, contractual arrangements that combine private operation with public financing is the most sustainable option in many countries. Availability of quality infrastructure is a key for the growth, economic prosperity and global integration of any country. Infrastructure has been relatively a neglected sector of the Indian economy, till the end of the X Plan.

\subsection{Why India Needs Widespread Use of PPPs?}

The performance of the infrastructure services is described as a mixed bag during the Eleventh Plan period. The total investment in infrastructure which includes roads, railways, ports, airports, electricity, telecommunications, oil, gas, pipelines and irrigation has increased from 5.7 per cent of Gross Domestic Product (GDP) in the base year of the Eleventh Plan to around 8.4 per cent in 2010-11.

\subsection{Prospects for the Twelfth Plan}

India needs to create infrastructure to the extent of $\$ 10$ trillion in three decades. It needs to invest $\$ 1$ trillion in the infrastructure sector, during the Twelfth plan period ending march 2017. It is necessary to bring new paradigm of thinking for infrastructure development. The Planning Commission has explored two alternative targets for economic growth in the Twelfth Plan, the first is a restatement of the Eleventh Plan target of 9.0 per cent growth, which could not be achieved. The second is an even higher target of 9.5 per cent average growth for the Twelfth Five Year Plan. The growth rate of 9.5 per cent requires much faster growth in electricity, gas and water supply sectors. The Twelfth Plan sets an ambitious target of one lakh MW in power generation. Against Eleventh Plan investment for infrastructure, projected at rupees 5,14,040crores of total infrastructure investment, Twelfth Plan envisages an investment of fifty lakh crores in five years (Planning Commission, 2011). 
Overemphasis on Public Private Partnership: Out of total investment of fifty lakhs crore over a period of five years, private sector is expected to provide 25 lakh crore. Government looks to be withdrawing from infrastructure sectors in Twelfth Five Year Plan and lays overemphasis on public private partnership. Planning Commission rides high on the success of PPP model in road transport and modernization of airports. The PPP based development needs to be encouraged wherever feasible.

\subsection{Advantages of PPPs for India}

India needs more widespread use of PPPs to meet infrastructure requirements. PPPs become all the more important for India because of the following reasons:

- Government doesn't have the financial room to develop infrastructure, hence PPP projects are indispensible.

- Private investments needs access to long term funds.

- Banks are not in a position to fund infrastructure because of asset liability mismatch. Sunil Mittal in his article "Can He Bring Back The Spark" in Economic Times (17the May, 2014) has pointed out that it is very important to encourage and support PPPs as, the gross non-performing assets as percentage of advances are increasing, thus making it more difficult for flow of funds of banks for infrastructure development.

- Privatization is not the best form in a country like India.

- PPPs will help to reduce the backwardness in infrastructure and put India on par with developed countries.

- The Government of India is promoting PPPs as an effective tool for bringing private sector efficiencies in creation of economic and social infrastructure assets and for delivery of quality public services.

\subsection{Public-Private Partnership Initiative in India}

By end March 2014, there were over 1300 projects in the infrastructure sector with a total project cost (TPC) of Rs. 694,040 crore. These projects are at different stages of implementation i.e. bidding, construction and operational. Some fo the important PPP initiatives in India re given below.

- Under the Viability Gap Funding (VGF) scheme for projects - 178 projects have been granted approvals with a TPC of Rs. 88,697 crore and VGF support of Rs. 16,894 crore, of which Rs. 1455 crore has been disbursed. 
72 | MANTHAN: Journal of Commerce and Management, Vol. 2, Issue 2

- The India Infrastructure Project Development Fund (IIPDF) was launched in December 2007 to facilitate quality project development for PPP projects and ensure transparency in procurement of consultants and projects.

- The National PPP Capacity Building Programme was launched in December 2010, and has been rolled out in sixteen states and two central training institutes i.e. the Indian Maritime University and Lal Bahadur Shastri National Academy of Administration. The roll out in the respective institutes commenced in 2011-12. Till March 2014, 160 training programmes have been conducted to train over 5000 public functionaries who deal with PPPs in their domain.

- The PPP online toolkit is a web-based resource that has been designed to help improve decision making for infrastructure PPPs in India and to improve the quality of infrastructure PPPs that are implemented.

- Many State Governments have institutionalised measures to encourage private sector engagement in creation of infrastructure delivery of services. Infrastructure Development and Enabling Acts have been developed by Andhra Pradesh, Bihar, Gujarat, and Punjab. PPP policies and guidelines to facilitate PPP projects have been notified by Karnataka, Haryana, Orissa, Assam, Goa, Madhya Pradesh and West Bengal. Other measures include development of sectoral policies for promoting PPPs, establishing nodal departments/PPP cells, establishing project development funds to supplement Government of India (GOI) grant, establishing panels of transaction advisers and developing standardized bid documents, sectoral templates and handbooks on PPPs. Awareness of schemes, guidelines initiatives and resource materials prepared is being created through PPP websites of Central and State Governments.

- The annual report of top fifteen infrastructure companies that have developed PPP format have been analysed to determine the present and future environment for PPPs.

\subsection{Infrastructure Companies that have adopted PPP format}

The companies selected for analysis are Larsen \& Toubro (L \& T), Jaiprakash Associates (JAL), LancoInfratech, Relience Infrastructure Limited, GMR Infrastructure, Punj Llyod Limited, IVRCL Infrastructure Limited, Hindustan Construction Company (HCC), NCC Limited, Gammon Limited, Simplex Infrastructure Limited, GVK Power \& Infrastructure Limited, IRB Infrastructure Developers, Patel Engineering Company, Gayatri Project Limited. The parameters which have been considered for analysis are profitability, rate of return and ability to repay debt. 


\subsection{Profitability}

Profitability of the selected companies have been analysed in terms of 'Profit before interest and tax' and 'earnings per share'.

Profit Before Interest and Tax (PBIT) (\%): PBIT is a well-known indicator of a company's profitability. It nulls the effect of the different capital structures and tax rates used by different companies, and hones in on the company's ability to profit. The companies whose PBIT is above 10 per cent are - Larsen \& Toubro (L \& T), Jaiprakash Associates (JAL), LancoInfratech, Relience Infrastructure Limited, GMR Infrastructure, Hindustan Construction Company (HCC), Patel Engineering Company, GVK Power \& Infrastructure Limited, and Gayatri Project Limited (Table 1). Gammon Ltd, earned PBIT in the year 2010-11 and posted an impressive profit of 69.21 per cent but is now buffeted with some headwinds as net profits are declining. Other companies earned PBIT less than 10 per cent.

Earnings Per Share (EPS): This measures the profit available to the equity shareholders on a per share basis. Other things remain constant if EPS has increased over the years, it means that firm's profitability has improved. The EPS of GMR Infrastructure Ltd, Reliance Infrastructure Ltd and IRB Infrastructure Developers Ltd, has shown upward trend (Table 2), while for other companies has declined overtime. The reasons for declining EPS are companies repaying their long term debts, recession, high interest cost, taxes, high labour charges, escalation in the cost of projects due to delays, time and cost overruns.

\subsection{Return on Capital Employed (ROCE)}

This ratio provide insight into how efficiently the long - term funds, supplied by the lenders and owners of the firm are being used (Table 3). This ratio has gone up for Reliance Infrastructure Limited, GMR Infrastructure Ltd, Hindustan Construction Company Limited and IRB Infrastructure Developers Ltd. Reliance Infrastructure Ltd has increased its borrowings the company's share market prices have witnessed a positive rise in the period 2014. Other companies have experienced a decline in ROCE, because of global recession.

\subsection{Ability to Repay Debt Ratio}

Debt-Equity Ratio $(D / E)$ : It is the ratio of the amount invested by outsiders to the amount invested by the owners of business. The D/E ratio indicates the margin of safety to the creditors. 
74 | MANTHAN: Journal of Commerce and Management, Vol. 2, Issue 2

Table 1: Profit before Interest and Taxes of Fifteen Infrastructure Companies for the year 2008-2014

\begin{tabular}{|c|c|c|c|c|c|c|c|}
\hline & & & & & \multicolumn{3}{|c|}{ (Margin \%) } \\
\hline $\begin{array}{l}\text { Year } \\
\text { Company } \\
\text { Name }\end{array}$ & $\begin{array}{l}\text { Mar } \\
2008\end{array}$ & $\begin{array}{l}\text { Mar } \\
2009\end{array}$ & $\begin{array}{l}\text { Mar } \\
2010\end{array}$ & $\begin{array}{l}\text { Mar } \\
2011\end{array}$ & $\begin{array}{l}\text { Mar } \\
2012\end{array}$ & $\begin{array}{l}\text { Mar } \\
2013\end{array}$ & $\begin{array}{l}\text { Mar } \\
2014\end{array}$ \\
\hline $\begin{array}{l}\text { Larsen \& Toubro } \\
\text { Limited }\end{array}$ & 11.97 & 11.14 & 12.41 & 11.18 & 10.24 & 8.90 & 10.04 \\
\hline $\begin{array}{l}\text { Jaiprakash Associates } \\
\text { Limited }\end{array}$ & 22.50 & 23.61 & 19.01 & 18.56 & 21.53 & 19.23 & 18.58 \\
\hline $\begin{array}{l}\text { LancoInfratech } \\
\text { Limited }\end{array}$ & 18.89 & 12.85 & 13.44 & 12.62 & 6.31 & 10.96 & 18.56 \\
\hline $\begin{array}{l}\text { Reliance Infrastructure } \\
\text { Limited }\end{array}$ & 4.45 & 1.78 & 5.15 & 3.02 & 12.66 & 9.87 & 12.46 \\
\hline $\begin{array}{l}\text { GMR Infrastructure } \\
\text { Limited }\end{array}$ & 84.52 & 78.68 & 36.71 & 28.70 & 20.26 & 24.11 & 31.91 \\
\hline PunjLlyod Limited & 9.32 & 9.39 & 10.50 & 6.95 & 5.34 & 5.51 & 5.88 \\
\hline $\begin{array}{l}\text { IVRCL Infrastructure } \\
\text { Limited }\end{array}$ & 9.90 & 9.29 & 10.85 & 9.63 & 6.02 & 5.44 & 1.64 \\
\hline $\begin{array}{l}\text { Hindustan } \\
\text { Construction Company }\end{array}$ & 10.30 & 9.65 & 9.41 & 9.42 & 6.90 & 5.07 & 11.40 \\
\hline NCC Limited & 11.18 & 9.58 & 11.35 & 10.27 & 5.88 & 6.47 & 5.02 \\
\hline Gammon Limited & 7.02 & 7.19 & 7.35 & 2.93 & 6.35 & -0.14 & -9.94 \\
\hline $\begin{array}{l}\text { Simplex Infrastructure } \\
\text { Limited }\end{array}$ & 8.74 & 7.18 & 7.86 & 7.81 & 5.95 & 5.76 & 6.85 \\
\hline $\begin{array}{l}\text { GVK Power \& } \\
\text { Infrastructure Limited }\end{array}$ & 11.59 & - & 32.01 & 16.89 & 28.52 & 24.13 & 33.60 \\
\hline $\begin{array}{l}\text { IRB Infrastructure } \\
\text { Developers Limited }\end{array}$ & -14.37 & $\begin{array}{c}- \\
12.88\end{array}$ & -22.10 & 12.61 & 9.80 & 9.60 & 9.06 \\
\hline $\begin{array}{l}\text { Patel Engineering } \\
\text { Company Limited }\end{array}$ & 17.47 & 13.12 & 15.46 & 13.19 & 10.96 & 12.10 & 11.11 \\
\hline $\begin{array}{l}\text { Gayatri Projects } \\
\text { Limited }\end{array}$ & 12.24 & 9.41 & 10.52 & 11.87 & 9.46 & 11.59 & 13.20 \\
\hline
\end{tabular}

Source: *Annual Reports of above mentioned companies

*Dion Global Solutions Limited 
Table 2: Earning Per Share of Fifteen Infrastructure Companies for the years 2008-2014

(in Rs.)

\begin{tabular}{|l|c|c|c|c|c|c|c|}
\hline Year & $\begin{array}{c}\text { Mar } \\
\mathbf{2 0 0 8}\end{array}$ & $\begin{array}{c}\text { Mar } \\
\mathbf{2 0 0 9}\end{array}$ & $\begin{array}{c}\text { Mar } \\
\mathbf{2 0 1 0}\end{array}$ & $\begin{array}{c}\text { Mar } \\
\mathbf{2 0 1 1}\end{array}$ & $\begin{array}{c}\text { Mar } \\
\mathbf{2 0 1 2}\end{array}$ & $\begin{array}{c}\text { Mar } \\
\mathbf{2 0 1 3}\end{array}$ & $\begin{array}{c}\text { Mar } \\
\mathbf{2 0 1 4}\end{array}$ \\
\hline Larsen \& Toubro Limited & 74.35 & 59.45 & 72.66 & 65.01 & 72.77 & 79.8 & 59.26 \\
\hline $\begin{array}{l}\text { Jaiprakash Associates } \\
\text { Limited }\end{array}$ & 5.20 & 7.58 & 8.04 & 5.49 & 4.83 & 2.26 & 1.87 \\
\hline LancoInfratech Limited & 9.00 & 11.91 & 2.02 & 1.16 & 0.48 & 0.06 & -3.99 \\
\hline $\begin{array}{l}\text { Reliance Infrastructure } \\
\text { Limited }\end{array}$ & 45.36 & 50.39 & 47.03 & 40.42 & 76.06 & 76.03 & 60.38 \\
\hline $\begin{array}{l}\text { GMR Infrastructure } \\
\text { Limited }\end{array}$ & 0.34 & 0.54 & 0.04 & 0.15 & 0.31 & 0.14 & 0.43 \\
\hline PunjLlyod Limited & 7.30 & 10.58 & 11.06 & 0.37 & 1.74 & 0.59 & 0.24 \\
\hline $\begin{array}{l}\text { IVRCL Infrastructure } \\
\text { Limited }\end{array}$ & 15.77 & 16.93 & 7.91 & 5.91 & 0.68 & -3.31 & - \\
\hline $\begin{array}{l}\text { Hindustan Construction } \\
\text { Company }\end{array}$ & 4.24 & 4.89 & 2.69 & 1.17 & -3.66 & -2.27 & 1.33 \\
\hline NCC Limited & 7.08 & 6.72 & 9.07 & 6.37 & 1.40 & 2.44 & 1.58 \\
\hline Gammon Limited & 10.18 & 16.19 & 9.39 & 8.73 & 6.41 & - & - \\
\hline $\begin{array}{l}\text { Simplex Infrastructure } \\
\text { Limited }\end{array}$ & 18.21 & 24.42 & 24.78 & 24.91 & 18.03 & 12.09 & 12.25 \\
\hline $\begin{array}{l}\text { GVK Power \& } \\
\text { Infrastructure Limited }\end{array}$ & 0.64 & 0.15 & 0.14 & 0.43 & -0.05 & -0.15 & -0.15 \\
\hline $\begin{array}{l}\text { IRB Infrastructure } \\
\text { Developers Limited }\end{array}$ & 0.18 & 1.73 & 1.68 & 2.71 & 4.99 & 5.64 & 8.67 \\
\hline $\begin{array}{l}\text { Patel Engineering } \\
\text { Company Limited }\end{array}$ & 24.74 & 29.22 & 18.74 & 14.64 & 8.65 & 7.05 & 3.28 \\
\hline Gayatri Projects Limited & 39.64 & 41.19 & 48.04 & 52.06 & 19.18 & 20.87 & 15.75 \\
\hline
\end{tabular}

Source: *Annual Reports of above mentioned companies

*Dion Global Solutions Limited 
76 | MANTHAN: Journal of Commerce and Management, Vol. 2, Issue 2

Table 3: Return on Capital Employed of Fifteen Infrastructure Companies for the years 2008-2014

\begin{tabular}{|l|c|c|c|c|c|c|c|}
\hline \multicolumn{1}{|c|}{ Year } & $\begin{array}{c}\text { Mar } \\
\mathbf{2 0 0 8}\end{array}$ & $\begin{array}{c}\text { Mar } \\
\mathbf{2 0 0 9}\end{array}$ & $\begin{array}{c}\text { Mar } \\
\mathbf{2 0 1 0}\end{array}$ & $\begin{array}{c}\text { Mar } \\
\mathbf{2 0 1 1}\end{array}$ & $\begin{array}{c}\text { Mar } \\
\mathbf{2 0 1 2}\end{array}$ & $\begin{array}{c}\text { Mar } \\
\mathbf{2 0 1 3}\end{array}$ & $\begin{array}{c}\text { Mar } \\
\mathbf{2 0 1 4}\end{array}$ \\
\hline $\begin{array}{l}\text { Larsen \& Toubro } \\
\text { Limited }\end{array}$ & 26.72 & 24.14 & 22.49 & 21.95 & 20.66 & 20.02 & 17.18 \\
\hline $\begin{array}{l}\text { Jaiprakash Associates } \\
\text { Limited }\end{array}$ & 9.77 & 9.53 & 8.61 & 9.52 & 10.87 & 8.2 & 7.29 \\
\hline LancoInfratech Limited & 15.39 & 16.9 & 15.57 & 10.47 & 8.74 & 7.84 & -3.97 \\
\hline $\begin{array}{l}\text { Reliance Infrastructure } \\
\text { Limited }\end{array}$ & 6.56 & 6.22 & 8.08 & 6.29 & 11.07 & 8.53 & 7.90 \\
\hline $\begin{array}{l}\text { GMR Infrastructure } \\
\text { Limited }\end{array}$ & 1.46 & 2.05 & 0.81 & 2.09 & 3.38 & 3.47 & 2.07 \\
\hline PunjLlyod Limited & 11.92 & 12.42 & 11.75 & 5.41 & 8.28 & 8.41 & 9.19 \\
\hline $\begin{array}{l}\text { IVRCL Infrastructure } \\
\text { Limited }\end{array}$ & 17.12 & 17.99 & 18.64 & 14.85 & 11.63 & 5.96 & 2.34 \\
\hline $\begin{array}{l}\text { Hindustan Construction } \\
\text { Company }\end{array}$ & 11.57 & 10.73 & 9.24 & 8.55 & 8.28 & 5.58 & 12.03 \\
\hline NCC Limited & 17.71 & 16.18 & 16.36 & 10.91 & 9.78 & 11.15 & 9.71 \\
\hline Gammon Limited & 14.64 & 13.52 & 12.80 & 10.38 & 10.46 & 2.19 & -4.41 \\
\hline $\begin{array}{l}\text { Simplex Infrastructure } \\
\text { Limited }\end{array}$ & 17.45 & 17.24 & 16.40 & 14.66 & 11.28 & 9.63 & 9.75 \\
\hline $\begin{array}{l}\text { GVK Power \& } \\
\text { Infrastructure Limited }\end{array}$ & 6.15 & - & 1.27 & 0.33 & 0.82 & 1.42 & 1.77 \\
\hline $\begin{array}{l}\text { IRB Infrastructure } \\
\text { Developers Limited }\end{array}$ & 2.46 & 4.06 & 2.63 & 4.89 & 8.53 & 14.74 & 14.73 \\
\hline $\begin{array}{l}\text { Patel Engineering } \\
\text { Company Limited }\end{array}$ & 14.49 & 18.39 & 13.30 & 11.97 & 11.62 & 12.59 & 9.49 \\
\hline $\begin{array}{l}\text { Gayatri Projects } \\
\text { Limited }\end{array}$ & 15.38 & 17.39 & 15.34 & 13.77 & 11.82 & 12.13 & 12.22 \\
\hline
\end{tabular}

Source: *Annual Reports of above mentioned companies

*Dion Global Solutions Limited

Interest Coverage Ratio: This ratio measures the debt servicing capacity of a firm in so far as fixed interest on long - term loan is concerned. 
The solvency ratios of fifteen infrastructure companies that have adopted PPP format is presented in Table 4. For companies like Larsen \& Toubro Ltd, Jaiprakash Associates Ltd and Gayatri Projects Ltd, during recession D/E ratio had increased, but in 2014 it is back to stable level.

Table 4: Debt Equity Ratio of Fifteen Infrastructure Companies for the years 20082014

\begin{tabular}{|l|c|c|c|c|c|c|c|}
\hline \multicolumn{1}{|c|}{ Year } & $\begin{array}{c}\text { Mar } \\
\mathbf{2 0 0 8}\end{array}$ & $\begin{array}{c}\text { Mar } \\
\mathbf{2 0 0 9}\end{array}$ & $\begin{array}{c}\text { Mar } \\
\mathbf{2 0 1 0}\end{array}$ & $\begin{array}{c}\text { Mar } \\
\mathbf{2 0 1 1}\end{array}$ & $\begin{array}{c}\text { Mar } \\
\mathbf{2 0 1 2}\end{array}$ & $\begin{array}{c}\text { Mar } \\
\mathbf{2 0 1 3}\end{array}$ & $\begin{array}{c}\text { Mar } \\
\mathbf{2 0 1 4}\end{array}$ \\
\hline $\begin{array}{l}\text { Larsen \& Toubro } \\
\text { Limited }\end{array}$ & 0.38 & 0.53 & 0.37 & 0.29 & 0.33 & 0.27 & 0.34 \\
\hline $\begin{array}{l}\text { Jaiprakash Associates } \\
\text { Limited }\end{array}$ & 2.12 & 2.10 & 2.17 & 2.02 & 1.31 & 1.52 & 1.69 \\
\hline LancoInfratech Limited & 0.35 & 0.72 & 0.89 & 1.12 & 0.94 & 1.14 & 2.12 \\
\hline $\begin{array}{l}\text { Reliance Infrastructure } \\
\text { Limited }\end{array}$ & 0.49 & 0.7 & 0.29 & 0.23 & 0.49 & 0.51 & 0.66 \\
\hline $\begin{array}{l}\text { GMR Infrastructure } \\
\text { Limited }\end{array}$ & 0.09 & 0.07 & 0.44 & 0.33 & 0.38 & 0.52 & 0.71 \\
\hline PunjLlyod Limited & 0.57 & 1.13 & 0.98 & 0.93 & 1.01 & 1.19 & 1.27 \\
\hline $\begin{array}{l}\text { IVRCL Infrastructure } \\
\text { Limited }\end{array}$ & 0.67 & 0.77 & 0.87 & 1.06 & 1.09 & 1.15 & 2.44 \\
\hline $\begin{array}{l}\text { Hindustan Construction } \\
\text { Company Limited }\end{array}$ & 1.87 & 2.35 & 1.66 & 2.28 & 2.62 & 3.93 & 3.64 \\
\hline NCC Limited & 0.57 & 0.74 & 0.68 & 1.04 & 0.85 & 0.83 & 0.91 \\
\hline Gammon Limited & 0.39 & 0.88 & 0.77 & 1.13 & 1.20 & 1.92 & 4.15 \\
\hline $\begin{array}{l}\text { Simplex Infrastructure } \\
\text { Limited }\end{array}$ & 1.02 & 1.39 & 1.34 & 1.54 & 1.74 & 2.07 & 2.08 \\
\hline $\begin{array}{l}\text { GVK Power \& } \\
\text { Infrastructure Limited }\end{array}$ & - & - & 0.04 & 0.04 & 0.16 & 0.17 & 0.19 \\
\hline $\begin{array}{l}\text { IRB Infrastructure } \\
\text { Developers Limited }\end{array}$ & 0.01 & 0.10 & 0.48 & 0.88 & 1.19 & 0.72 & 1.06 \\
\hline $\begin{array}{l}\text { Patel Engineering } \\
\text { Company Limited }\end{array}$ & 0.78 & 1.04 & 1.24 & 1.42 & 1.33 & 1.70 & 2.03 \\
\hline Gayatri Projects Limited & 2.52 & 1.67 & 2.20 & 2.81 & 1.85 & 2.13 & 1.97 \\
\hline
\end{tabular}

Source: *Annual Reports of above mentioned companies

*Dion Global Solutions Limited 
For LancoInfratech Ltd, the company has gone for fresh borrowings, availed at lower rate of interest, and thus has tried to recover its financial position by lowering its interest obligation. Reliance Infrastructure Ltd and GMR Infrastructure Ltd, have increased borrowings for March ending 2014, but the interest coverage has been handled. For Punj Lloyd Ltd, IVRCL Infrastructure Ltd, HCC and NCC Ltd, have increased their debt margin, but have experienced a drop in profit margin owing to commodity price pressure, high labour charges and ineligibility to pass on escalations in the costs of infrastructure projects.

For Gammon Ltd., the company is reported to be weighed down with a few woes-low-margin legacy orders, working capital needs which increased debt, depressed the order intake and earnings. However, the company seems to be confident to face all challenges. Simplex Infrastructure Ltd has handled its debt equity proportion by increase of 1.06 per cent, while GVK Power \&Infrastructure Ltd has gone in for new infrastructure projects having an order book of around Rs. 12000 crores. It has increased its debts to meet the increasing finance requirements; as a result interest coverage ratio has declined (Table 5). IRB Infrastructure Developers, to finance new infrastructure projects, has increased its debt ratio to 1.06:1 for March ending 2014. IRB Infrastructure reported a stellar performance on all fronts. For Patel Engineering, its core Engineering Procurement and Construction (EPC) is currently facing headwinds, as large projects are facing delays. The longer gestation nature of its order book, has increased its debt level. Gayatri Projects Ltd, because of delays in government sanctions, experienced decline in profit margin, as a result there was increase in debts for the year 2011. The company has handled their debts and the D/E ratio has dropped to 1.97 for March 2014 from 2.5 in March 2008.

\subsection{The Road Ahead}

The future prospects and the road ahead for the companies under study have been analysed below.

Larsen \& Turbo: A proxy to India's infrastructure story continues to hold a unique place in the Indian Engineering and Construction space as a diversified and leading engineering player. The company is witnessing good transaction on the international front and expects 25per cent revenue growth.

Jaiprakash Associates: Order book stands at Rs.55,000crore. It has two contracts worth Rs. 2079 crore to construct hydroelectric projects, to be jointly implemented by Government of Bhutan and Indian Government. 
LancoInfratech: The group has been on a growth trajectory having a total order of book value of Rs.31,016crore (2014) of which about 90 per cent of its revenue is from thermal and solar power.

Table 5: Interest Coverage of Fifteen Infrastructure Companies for the year 20082014

\begin{tabular}{|l|c|c|c|c|c|c|c|}
\hline \multicolumn{1}{|c|}{ Year } & $\begin{array}{c}\text { Mar } \\
\mathbf{2 0 0 8}\end{array}$ & $\begin{array}{c}\text { Mar } \\
\mathbf{2 0 0 9}\end{array}$ & $\begin{array}{c}\text { Mar } \\
\mathbf{2 0 1 0}\end{array}$ & $\begin{array}{c}\text { Mar } \\
\mathbf{2 0 1 1}\end{array}$ & $\begin{array}{c}\text { Mar } \\
\mathbf{2 0 1 2}\end{array}$ & $\begin{array}{c}\text { Mar } \\
\mathbf{2 0 1 3}\end{array}$ & $\begin{array}{c}\text { Mar } \\
\mathbf{2 0 1 4}\end{array}$ \\
\hline Larsen \& Toubro Limited & 28.57 & 13.09 & 11.17 & 9.99 & 10.39 & 7.57 & 7.21 \\
\hline $\begin{array}{l}\text { Jaiprakash Associates } \\
\text { Limited }\end{array}$ & 3.61 & 3.67 & 2.12 & 1.98 & 1.73 & 1.37 & 0.98 \\
\hline LancoInfratech Limited & 11.76 & 3.91 & 4.64 & 2.24 & 1.21 & 1.02 & -0.53 \\
\hline $\begin{array}{l}\text { Reliance Infrastructure } \\
\text { Limited }\end{array}$ & 3.41 & 3.65 & 5.18 & 5.50 & 5.40 & 2.96 & 2.80 \\
\hline $\begin{array}{l}\text { GMR Infrastructure } \\
\text { Limited }\end{array}$ & 4.08 & 5.47 & 1.07 & 1.18 & 1.71 & 1.02 & 0.63 \\
\hline PunjLlyod Limited & 4.01 & 3.55 & 3.15 & 1.20 & 1.16 & 1.04 & 1.02 \\
\hline $\begin{array}{l}\text { IVRCL Infrastructure } \\
\text { Limited }\end{array}$ & 5.04 & 3.53 & 3.40 & 2.58 & 1.09 & 0.80 & 0.20 \\
\hline $\begin{array}{l}\text { Hindustan Construction } \\
\text { Company }\end{array}$ & 2.09 & 1.59 & 1.65 & 1.31 & 0.74 & 0.61 & 1.15 \\
\hline NCC Limited & 4.58 & 3.28 & 3.55 & 3.71 & 1.14 & 1.24 & 1.01 \\
\hline Gammon Limited & 3.80 & 2.30 & 2.04 & 1.65 & 1.43 & 0.25 & -0.59 \\
\hline $\begin{array}{l}\text { Simplex Infrastructure } \\
\text { Limited }\end{array}$ & 2.81 & 2.94 & 3.74 & 3.32 & 1.61 & 1.31 & 1.26 \\
\hline $\begin{array}{l}\text { GVK Power \& } \\
\text { Infrastructure Limited }\end{array}$ & 16.28 & - & 38.00 & 0.62 & 0.98 & 0.80 & 0.90 \\
\hline $\begin{array}{l}\text { IRB Infrastructure } \\
\text { Developers Limited }\end{array}$ & 4.20 & 2.12 & 2.17 & 1.57 & 1.33 & 1.18 & 1.08 \\
\hline $\begin{array}{l}\text { Patel Engineering } \\
\text { Company Limited }\end{array}$ & 3.47 & 3.07 & 3.29 & 2.55 & 1.67 & 1.75 & 1.52 \\
\hline Gayatri Projects Limited & 23.64 & 10.19 & 4.99 & 3.78 & 2.37 & 2.47 \\
\hline
\end{tabular}

Source: *Annual Reports of above mentioned companies

*Dion Global Solutions Limited

GMR Infrastructure: It has embarked on a wide range of projects; to name a few, 800 MW island power at Singapore, $25 \mathrm{MW}$ solar project at Gujarat, started construction for 
the $1370 \mathrm{MW}$, Chhatisgarh thermal power project and 2.1 MW wind power project at Gujarat.

Punj Llyod Ltd: The group's order book is mainly dominated by the infrastructure (37.8\%) and pipeline (25.6\%) segments. The company linked pact with Qatar Solar Technologies to set up first Polysilicon plant worth US \$1 billion in Qatar and a thermal power contract worth Rs.1,195crore West Bengal.

IVRCL Infrastructure Ltd: The company's order book stands at Rs.21,550crore (March 2014) and its infrastructure projects are diversified across six segments. It has reported 1.6 per cent growth annually.

HCC Ltd: The order book consists of an assortment of projects, such as hydro (42\%), transport (22\%), water $(20 \%)$, nuclear and others $(16 \%)$. It has been awarded a contract worth Rs.18.43 billion to construct Tehri pumped storage plant in Uttarakhand.

NCC Ltd: The group has project aggregating to Rs.629 crore (March 2014), spread across nine verticals. It is in the process of winning EPC orders worth Rs. 5000 crore, from Nelcast power project.

Gammon Ltd: It has a strong order book position around Rs.15100 crore as on (March,2014), and has a stimulating basket of ongoing infrastructure projects worth Rs.95,369crore in the road, port and the energy sectors.

Simplex Infrastructure Ltd: The largest civil construction and engineering contractor completed 2400 projects in India and abroad.It recently completed the $11.5 \mathrm{Km}$ longest elevated expressway road in Mumbai, Chennai, Bangladesh and Middle East Countries.

GVK Power and Infrastructure Ltd: Its income has increased by 7 per cent to Rs.1,943.19crore (2014) from Rs.1815.82crore in the previous year. It power assets contributed an income of 88.15 per cent. Other segments that contributed to its income were the Mumbai and Bangalore airports.

IRB Infrastructure Developers: The company has bagged the first mega project floated by NHAI for which the approximate cost will be Rs.3500 crore. IRB has an order backlog of Rs. 11,500 crore and is well placed to lead the infrastructure sector.

Patel Engineering Company: The major part of its order inflow is in the road segment. It has invested equity of Rs. 270 crore (march 2014) in its power ventures.

Gayatri Projects Ltd: The company has taken up projects for execution from Nercas. Its clientele includes NHAI, state governments, special purpose vehicles (SPV) constituted by the company for execution of built-operate-transfer (BOT) projects of public sector undertakings and private sector companies.

In the above analysis of top fifteen infrastructure companies which have adopted PPP format, these companies have huge infrastructure investment which involves large sum of money being invested in huge projects which are time consuming with long 
gestation period the companies reserves and borrowings are strained for a certain period, but consequently on successful execution the companies have recorded an increase in their profit margin.

As per $12^{\text {th }}$ Plan projections of investment of $\$ 1$ trillion in infrastructure sector, 50 per cent is expected to come from private sector. Economic Survey 2013-14 (p.10) points out that from the infrastructure development perspective of PPP projects, important issues like delays in regulatory approvals, problems in land acquisition, environmental clearances need immediate attention.

\subsection{Factors constraining Private Investment}

Different factors constraining private investment into infrastructure through PPP route are as listed below.

- Most of the PPP projects are getting delayed by three-four years. There are delays due to land acquisition as a result, there is 15 to 20 per cent cost escalation.

- The Land Acquisition Rehabilitation and Resettlement Act has increased cost dramatically and needs a relook.

- There are many projects that are stuck at the 90-95 per cent marks and need focused resolution. They need swift clearance.

- Many foreign firms are wary of investing in India, they feel there are too many permissions, too many stoppages and therefore they cannot plan the project and the cost in an accurate manner.

- By end of 2013-14, thirteen projects at a total project cost of Rs.16000 crore received no financial bids. Many infrastructure majors GMR and GVK are walking out of mega projects, worth over Rs.10,000crores as they are facing financial stress.

- India has awarded 758 PPP projects worth Rs. 4 lakh crore but there is no central law to govern PPPs.

- Corruption is an important area that needs to be addressed. The UN office on Drugs and Crime (UNODC) conducted a survey of 400 private sector and government officials to assess the ground realities on corruption in PPPs. The survey's findings, state that 42 per cent of firms feel roads and power are the sectors most prone to corruption. 87 per cent of private players said that bidding norms and tender criteria were rigged to suit certain bidders to which over 44 per cent of personnel agreed.

- Infrastructure projects in today's environment can only become viable if they get lowcost-funds. As construction costs have gone up, while traffic revenues have dipped. Private companies that expanded aggressively in the capital intensive infrastructure 
power sector are overloaded with debt and have burnt their fingers due to slow down in projects under execution and lower than expected returns on operational assets. Companies like GVK, GMR, Lanco have put on block projects and subsidiaries to reduce stress.

- Prasad (2013) lists the following problems experienced by PPP projects - Reliance Power cannot operate its newly built 2400 MW Somalkot plant as gas is not available, work on Tilaiya UMPP could not be started because government has still not handed over the land for the project. The Krishnapatnam UMPP has been stalled after price of Indonesian coal escalated. R-Infra bagged two of the biggest transmission projects in the country but could not start work due to delay in government clearance. These delays threaten giant projects.

\subsection{Suggestions for Future Rollout of PPPs}

- It is important to set realistic milestones for programmes rather than impose unattainable deadlines on underprepared organizations.

- It is equally important to build capacities of government agencies to administer complex contracts and deliver on critical obligation quickly.

- As recommended by various stakeholders, establishing independent regulatory and dispute settlement mechanisms for expeditious settlement of disputes or to deal with industry wide concerns needs to be fully explored.

- For PPPS to become a mainstream form of public service delivery, one crucial attitude in all stakeholders would be the unambiguous intention to honour their respective contractual obligations. A national programme for character building is perhaps, the only way to achieve this.

- In order to attract international investments, and also the best players, we have to prove that ours is a nation where justice is speedy and certain. One way to expedite decisions is to have special courts for infrastructure arbitration.

- Relooking at certain basic norms will help, say industry insiders. In roads, for example there are several areas in India where there has been no traffic growth, and hence, extending the 8-10 years given to break-even would help companies cover the increasing cost of construction.

- Most of the PPP projects are getting delayed by six months to three-four years, but are not getting the relief for the cost escalation. There should be relaxation of exit clauses of infrastructure projects, which is essential for liquidity and very important for a concessionaire, to unlock money and move to the next level. 
- The role of multiple agencies should be curtailed. With multiple agencies, there is always confusion over the role and responsibility of each. This increases project time, which results in increased holding costs.

- The Land Acquisition Rehabilitation and Resettlement Act has increased cost dramatically and needs a relook. There are many projects that are stuck at the 90-95 per cent, and need focused resolution, either because of a small land acquisition or a small environment approval. This needs swift clearance.

- The UNODC has flagged loopholes in Indian Law ability to control corruption and suggested that private partners in PPPs be designated as public officials to make them accountable under the Right to Information Act. This would bring such projects under the proposed laws to protect whistle blowers and guarantee service delivery to citizens. There is a need for legislation and procedures to address probity issues in PPPs. Most states do not have a legislation to regulate PPPs.

- As highlighted in Economic Survey 2013-14 "global experience indicates that PPPs work well when they combine the efficiency and risk assessment of the private sector with the public purpose of the government sector. They work poorly when rely on the efficiency and risk assessment of the government sector. India should be careful not to undertake PPPs that do not apportion risks and responsibility sensibly. Flexibility needs to be built into arrangements, so that the contract can be withdrawn and put up for rebid when the private party under performs.

- A cavalier approach of bank and financial investors to appraise projects, need to be replaced by a rigorous approach of banks and financial investors. A radical rethink in our financial systems is needed. Dedicated Infra Banks are needed, rather than the present banks whose sector specific limits are relatively small for large projects. The World Bank can also be convinced to be a partner or equity holder or a large deposit player.

- Though assets delivered through the PPP model and available for financing through securitization have risen, Indian infrastructure firms are hard pressed with the development of existing projects delayed and the attractiveness of new projects diminishing for private sectors funds and strategic operators. In order to provide a robust funding mechanism to the cash starved sector, the government and Securities Exchange Board of India (SEBI) will facilitate the securitization of projects assets through Infrastructure Business Trusts. The government will incentivize the creation of such trusts, which will help to raise long-term capital for the much needed sector. An infrastructure business trust will be set up as trust and registered with the market regulator. The regulator has proposed two categories of trusts. Category I trusts can 
raise funds through private placements from institutional investors only. These trusts can invest in multiple projects. The category II trust can raise funds from both local and foreign investors. However, it can invest only in commercially-operational projects. This trust route will help to boost infrastructure sector, and create a framework of fast-track, investment friendly and predictable PPPs to build largescale projects that are of vital importance for India to compete in global markets.

Future development of infrastructure will depend on how investment in infrastructure is facilitated. Such investment requires long-term funds with long pay back periods e.g. from insurance and pension funds as pointed in the Budget announced on $8^{\text {th }}$ March, 2015. The present Prime Minister Shri Narendra Modi and Finance Minister Shri Arun Jaitley are looking at alternative long-term and lower cost financing from pension funds like Rs. 7.5 lakh crore Employee Provident Fund (PPF). PPP is still a nascent concept in India and the ability of PPPs to become an efficient means of delivering public will depend on the intentions and spirit of all contracting parties, to honour their respective commitments.

\section{References}

Andres, Luis A, Guasch, J.luis, Thomas, Haven \& Vivien, Foster (2008). The Impact of Private Sector Participation in Infrastructure - Lights, Shadows and the Road Ahead. Washington DC: The World Bank. PPIAF, pp.223-237.

Cherian, Thomas. (2014, June 5). Honouring Contracts. The Economic Times, p.14.

Dasgupta,Yashddhara (2013, May 14). Infra Cos Seek Independent Body for PPP Projects. The Economic Times.

Delmon, Jeffrey. (2009). Private Sector Investment in Infrastructure - Project Finance, PPP Projects and Risk. Netherlands: Waters Kluwer, The World Bank. $2^{\text {nd }}$ ed.Ch1.

Dhoot, Vikas. (2013, June 3). PPP Projects Prone to Corruption. The Economic Times, p.14.

Dipesh, Dipu. (2013, June 3). Is PPP in Coal Mining, No Value for Money? The Economic Times.

Gala, Dipal. (2014, July 31). The Rising Infra Story. The Economic Times, p.13. 
Government of India, Ministry of Finance, Department of Economic Affairs, Economic Division (2014, July). Economic Survey 2013-14. Oxford University Press, p.213.

Government of India, Planning Commission, Twelfth Five Year Plan (2012-17), Volume I, para.1.75.

Government of India, Ministry of Finance, Department of Economic Affairs, (2011). Economic Survey 2010-11. New Delhi.

Government of India, Ministry of Finance, National Council of Applied Research 1996. The India Infrastructure Report Policy Imperatives for Growth and Welfare (Expert group on the commercialization of infrastructure projects). Volume-I, Executive Summary, June 1996.

Government of India, Planning Commission. (2011,October). Faster Sustainable and Inclusive Growth - An Approach to the Twelfth Five Year Plan 2012-17. Delhi: Government of India, Planning Commission.

Government of India, Planning Commission, (2008). Eleventh Five Year Plan 2007-12. Delhi: Government of India, Planning Commission, Volume III.

Kumar, Arun. (2014, June 20). Government plans trust route to boost infrastructure sector. The Economic Times, p.13.

Maharashtra Economic Development Council. (2007). Infrastructure in Maharashtra - A Compendium of Investment Projects. Maharashtra Economic Development Council.

Marin, Philipe. (2009). Public-Private Partnerships for Urban Water Utilities - A Review of Experiences in Developing Countries. Washington DC: The World Bank. PPIAF, pp.1-11.

Mittal, Sunil. (2014). Can he bring back the spark. The Economic Times. $17^{\text {th }}$ May.

Prasad, Rachita (2013, May 31). Tough road ahead for ADAG Infra projects. The Economic Times, pp.1,3. 
86 | MANTHAN: Journal of Commerce and Management, Vol. 2, Issue 2

Prasad, Chandrashekhar \& Rout, Himanshu Shekhar. (2013). Sixty Five Years of the Indian Economy - 1947-48 to 2012-13, New Delhi: New Century Publications July 2013, pp.435- 443.

Reserve Bank of India. (2010). Annual Report 2009-10, Supplement to RBI Bulletin.

Thakkar, Mitul (2014, August 5). Partnership Private Companies can make NTPC more efficient. The Economic Times.

WaltsHimangshu (2014, July 14). Jaitley Puts Infra on Growth Highway. The Economic Times, p.4.

\section{Websites}

www.pppindiadatabase.com

www.pppindia.com

www.moneycontrol.com 